\title{
Vision Based Fuzzy Control Approaches for Unmanned Aerial Vehicles
}

\author{
Miguel A. Olivares-Mendez ${ }^{1,2}$ Pascual Campoy ${ }^{2}$ \\ ${ }^{1}$ Automation Research Group, SnT - University of Luxembourg \\ ${ }^{2}$ Computer Vision Group, Universidad Politecnica de Madrid - Center of Automation and Robotics (CAR)
}

\begin{abstract}
This paper proposed the use of vision based Fuzzy control approaches for autonomous navigation tasks with Unmanned Aerial Vehicles (UAVs). It is shown the advantages of using RGB cameras as the sensor onboard UAVs and the advantages of using Fuzzy logic controllers. It is explained how to set a vision based system and how to define a Fuzzy controller for a general control approach. A specific software was design and used to develop and tune general Fuzzy control approaches. The "how-to" of this software is also explained in this paper. A methodology to how to design, developed and tune Vision based Fuzzy Control (VBFC) approaches in robotics is also presented. Furthermore, it is shown three different VBFC approaches for autonomous navigation developed using this methodology and software. Real experiments were done to validate the different approaches with different vertical takeoff and landing (VTOL) UAVs.
\end{abstract}

Keywords: Fuzzy Logic Control, Computer Vision, Vision Based Control, Unmanned Aerial Vehicles, Autonomous Navigation.

\section{Introduction}

The Unmanned Aerial Vehicles (UAVs) are getting popular in the last decade and they are not a robotics platform limited to military uses any more. We already witness today the successful arrival of UAVs on the market, thanks to the miniaturization of electronics, improving CPU power and reducing sensors' size and cost. Indeed, a wide range of basic to highly sophisticated UAVs are available from electronic stores to specialized companies focused on the development of these kinds of flying robots. The combination of different sensors with different types of control, together with the extreme cost reduction of using unmanned versus manned aerial vehicles, are leading to a great number of new UAV applications. Between all the possible sensor to use with UAVs and in general in robotics, the vision is most used. That is because of its low cost, related to other sensors, the similarities to the human vision, and the large possibilities of different algorithms to use. Regarding the control techniques, the Fuzzy control is one of the most used, because of the way of how to manage the uncertainty and imprecision on model identification and in the information acquired by the sensors.

In this paper it is proposed the use of vision based Fuzzy control in robotics, and more specific with UAVs. It is shown the advantages of using vision, and Fuzzy control, as well as how to set a general robotics vision based control approach. A general methodology to develop vision based Fuzzy control approaches is also explained. The validation of the explained vision based Fuzzy control technique is done by three different approaches of Fuzzy control system with different vision algorithms, different control objectives, and with three different UAVs.

The outline of the paper is organized as follows: In section 2 it is explained why vision is a good option as a sensor in robotics. Section 3 introduces the Fuzzy control as a technique for using the extracted information from the vision sensor in robotics. In section 4 it is shown the methodology to follow to developed vision based fuzzy control approaches for robotics. Section 5 presents the software developed for the design of Fuzzy controllers. The validation of the presented control method is done by three different VBFC approaches for autonomous navigation with unmanned aerial vehicles. The explanation of these approaches, the experiments done, and the different Unmanned aerial vehicles are presented in section 6. Finally, the concluding remarks and future work are presented in section 7 .

\section{Vision as the Sensor for Control Applications in Robotics}

Vision is an useful robotic sensor since it mimics the humans vision sense and allows to extract noncontact measurement from the environment. The use of vision with robots has a long history, starting with the work of Shirai and Inoue [1]. They describe how a visual feedback loop can be used to correct the position of a robot to increase task accuracy. Today vision systems are available from major vendors and they are highly integrated in robots systems. Typically visual sensing and manipulation are combined in a open-loop fashion, "looking" and then "moving". The accuracy of the results depends directly on the accuracy of the visual sensor, the robot end-effector and the controller. An alternative to increase overall accuracy of the system is to use a visual feedback control loop.

Visual servoing is no more than the use of vision 
at the lowest level, with simple image processing to provide reactive or reflexive behavior to servoposition a robotic system. Classical visual servo control was developed for serial-link robotic manipulators with the camera typically mounted on the end-effector, also called eye-in-hand.

Since the first works about visual servoing system, presented by Sanderson [2] at the decade of the 80 's, the progress in visual control of robots has been fairly slow. However, a huge amount of applications appeared in the last two decades, promoted by the increasing of the computing power, that allows the analysis of images at a sufficient rate to "servo" a robot manipulator.

The vision-based robot control using an eye-inhand system is classified into two groups: positionbased and image-based visual servoing, PBVS and IBVS respectively.

PBVS involves the reconstruction of the target pose with respect to the robot and leads to a Cartesian motion planning problem. This kind of control is based on the tri-dimensional information from the scene, so the geometric model of the object to track, and a calibrated model of the camera are needed. Then, the estimation of the position and orientation of the object are get. The sensitivity of PBVS design to camera calibration is particularly worrying when low quality camera are used.

In contrast, for IBVS the control task is defined in terms of image features. A controller is designed to maneuver the image features for a desire configuration. The original Cartesian motion planning problem is solved. The approach is inherently robust to camera calibration and target modeling errors reducing the computational cost. However, this configuration implies an extra complexity for the control design problem.

In particular, the dynamics of aerial robotic vehicles have proved difficulty to overcome and very few rigorous developments of IBVS control exist. Simulation tests of an helicopter stabilization with robust non-linear control are shown in [3]; helicopter kinematics was simplified as a Liouvillian system in [4]; path planning simulation examples involving a ground robot and a small autonomous helicopter are presented in [5]. On the other hand, there are more applications using the configuration of position-based visual control, as the trajectory tracking with a helicopter presented in [6], an autonomous landing helicopter [7], [8].

\section{Fuzzy Logic as the Control Technique for Vision Based approaches in Robotics}

The use of Fuzzy logic as a control technique was started by T. Yamakawa in 1989 [9]. The Fuzzy control became popular in Japan for the following 20 years, and then, its popularity was extended to Europe. As all the different kind of control techniques, this one has its advantages and disadvan- tages. Of course it is not the perfect solution for control all kind of processes. The principal useful cases to apply Fuzzy control are the management of qualitative, inexact or uncertain information, and the analysis of complex control processes by conventional quantitative techniques. The principal advantages of the Fuzzy control are, the linguistic control, using linguistic terms to adapt the human knowledge, the robust control, more than one single rule are involved in the control decision, so an error in a rule is not fatal, and how the multiple fuzzy rules and fuzzy controllers can manage complex nonlinear systems. As it is mentioned in the Fuzzy control book of Passino and Yurkovich [10], "Fuzzy control provides a formal methodology for representing, manipulating, and implementing a human's heuristic knowledge about how to control a system". The Fuzzy identification, modeling and control is presented in a gentle way by Takagi and Sugeno in [11].

The vision is a powerful and efficient sensor regarding its performance and its price relation. However, it is high dependent of the illumination conditions, and small changes could affect strongly in the precisions of the information acquired. That implies some uncertainty and imprecision in the data. Furthermore the complex non-linear systems used in robotics are difficult to identify and extract an exact model of the system to control. These peculiarities of a robotics vision based system make the Fuzzy control option to be an excellent choice. As L. Zadeh mentioned "As complexity rises, precise statements lose meaning and meaningful statements lose precision". Based on these remarks, when the system has noisy information, or no high precision and the model of the robotics system to control is difficult to identify, to use a Fuzzy control system is a high recommended option. Some examples of vision based Fuzzy control approaches in robotics are presented next. The system presented by Kok et al. for the context of Prey Capture, with a moving object as a passive 'prey' and a robot as a 'pursuer' in [12].In [13] it is proposed a fuzzy coordination control method among a group of distributed robots using only vision, keeping constant the relative distance and the angle. In [14] it is presented a vision tracking system to achieve high recognition performance under dynamic circumstances, using a fuzzy logic controller.

Regarding the specific case of use this control configuration for UAVs there are also some works done, such as the autonomous landing approach of a UAV with a kinect camera [15]. Or the development of a vision-based neuro-fuzzy controller for a two axes gimbal system mounted on a small UAV presented in [16]. 


\section{Implementation of a General Vision Based Fuzzy Control Approach}

To develop a new Fuzzy control approach is better to start with a simple configuration and to grow in complexity searching for more accurate behavior. The tuning process of the control approach can be done manually or using an optimization method, like neural networks, ANFIS, or Cross-Entropy. In the different approaches presented in section 6 all the tuning processes were done manually. We start setting the control approaches starting with a basic fuzzy control configuration with two or three inputs variables with reduced number of Fuzzy sets, and with a simple Fuzzy rules base set by heuristic rules or based on some human knowledges.

We fix the selection of the inference mechanism, the membership function shape and the defuzzzification method, but it is also something that could be changed during the tuning process. Then, if the behavior was not the expected one we firstly increase the number of sets in the variables, secondly the range of the variables has to be modified, and thirdly the size of the Fuzzy sets can be also modified. It is highly recommended to store data from different tests and then extract some statistics from how many times the different sets of all the variables have been used. Based on this data it is easy to divide a set that has been used too much in two or more sets, and in the other way, if there are two sets that have been used only few times, then they can be fused in only one set. Of course, the experiments must be diversified trying to cover most of the potential situations that the system can deal with. Finally, when the behavior is pretty close to the expected one, some minimal changes can be done in the rules outputs and in the exact location of the Fuzzy sets.

Here is presented the explained methodology by its different steps:

1. Define the problem to solve. What do you want to control?

2. Select the sensor: In the presented case it is the vision

- Select the characteristics to extract from the environment

- Define the system constraints: The camera resolution needed, and the minimum framerate/frequency to close the control loop

- Select the vision algorithm that gives the best performance based on the system constraints

3. Define the number of inputs and outputs of our system

4. Select the range of the variables based on the sensor

5. Choose the shape of the variables membership function
6. Select the inference mechanism and the defuzzification method

7. Define the number of fuzzy sets of each variable

8. Define a basic rule base based on heuristic information or human knowledge

9. Define the location and size of the Fuzzy sets

10. Test, test, and test

- If works with some problems Then goto 7

- If works but need minimum adjustments Then goto 9

\section{MOFS: A Fuzzy Control Design Software}

The design, development and implementation of all the Fuzzy control approaches presented in this work were done using an own developed $\mathrm{C}++$ library. This library is the Miguel Olivares' Fuzzy Software (MOFS), and nowadays it is also available for the Robotics Operative System (ROS) [17]. The library was designed by classes for each part of a Fuzzy logic controller, making it easier for future updates.There are different classes for variables, rules, membership function shape and defuzzification modes. Currently the library is adapted to design Mandami Fuzzy controllers, with no limitation on the number of inputs, and a single output. The membership function shape can be selected between triangular an trapezoidal. Two different inference mechanisms are implemented, the product and the sum. The defuzzification method implemented is the height of weights.

In this software it has been taken into account the possibility to define a symmetric and non-symmetric membership function for each variable. A symmetric membership function is defined by having a common size for all the fuzzy sets of this variable. A non-symmetric membership function is defined by non-equal sizes for the fuzzy sets of this variable. It has been proved in [18], [19], [20] that a special definition of the quantity and size of the fuzzy sets in the different variables can improve the behavior of a Fuzzy controller.

The MOFS also includes a supervised learning algorithm. It is based on the idea of the synapses weight of the brain neurons. Each rule has a assigned weight value. Initially all the weights are set with a default value (0.5), and it will change depending if the control output is equal to the user decision or not. There is a flag definition to activate/deactivate the learning algorithm. Once the learning algorithm is activated the user has to command the vehicle or system. In each situation evaluation the Fuzzy control checks the situation and gives an output based on the rules' base, this linguistic value is compared to the user's output. If both outputs belong to the same linguistic tags, the weight of the rules involved in this evaluation will be increased by the membership function value (belongs to $(0,1))$ divided by three. If the outputs do not belong to the same linguistic tag the weight 
value of all the rules involved will be decreased by a constant value equal to 0.3 . Once the weight of a rule goes below zero, the rule get the output of the linguistic value with higher membership function value of the user decision. In that way the controller modify its rules' base based on the user decisions, adapting its behavior to the different users, as is presented in [21].

\section{Fuzzy Control Approaches}

Here it is presented a summary of different Fuzzy control approaches for a set of applications and the different UAVs used. In all the cases real experiments are presented.

\subsection{Unmanned Aerial Vehicles}

In this work have been used three different UAVs. The first one was an electric powered helicopter of 1.7 meters of length. It allows us to carry $4 \mathrm{~kg}$ of weight. It was equipped with a VIA nano-itx CPU of $1.5 \mathrm{GHz}$ and fire-wire cameras with a pixels resolution of $320 x 240$. With this system configuration a simple Lucas-Kanade feature detection with optical flow algorithm [22] used to work at 8 frames per second.

After some time, the quadrotors appear on the research scene. These new UAVs allow us to do experiments easily, but with a very reduced payload. We start with a Pelican from AscTec. It can lift $650 \mathrm{gr}$ and was equipped with a Atom board of 1.6 $\mathrm{GHz}$. Better cameras were also available in this time. A USB 2.0 camera was installed in this UAV with a resolution of $640 x 480$. This system allows us to run the same vision algorithm used in the previous helicopter at 15 frame per seconds with the new resolution. Finally, we also used a low cost quadrotor version, the Ar.Drone parrot. The payload of this quadcopter is just less than $100 \mathrm{gr}$, and no CPU onboard was installed. The image capture by the $1280 x 720$ onboard camera was sent to an i5 2.0GHz laptop by WiFi, processes on it and sent back the command for the control purpose. In this case the common vision algorithm runs at 20 frames per seconds, but the WiFi communication and low range antennas introduce some delays in the image transmissions.

\subsection{Autonomous Landing}

A VTOL can land easier than a fixed wings, but it is required to keep the lateral and longitudinal position stable to go down. To rely on the GPS signal is not always possible because of the accuracy of the measures or the lack of signal (between buildings the reflexion effects increase the error), or because the UAV needs to land in a not static platform, like a ship or a boat. Here it is presented a vision based Fuzzy control approach for autonomous landing of a helicopter. In this case the camera is looking downward. The vision algorithm used is the $3 \mathrm{D}$ estimation based on homographies. The frameto-frame homography is estimated using matched points and robust model fitting algorithms. For it, the pyramidal Lucas-Kanade optical flow [22] on corners detected using the method of Shi and Tomasi [23] is used to generate a set of corresponding points, then, a RANSAC [24] algorithm is used to robustly estimate projective transformation between the reference object and the image. Here it is used a monochrome camera with a resolution of $640 \times 480$ and a known helipad.

The control system approach is composed by three Fuzzy controllers working in parallel, managing the longitudinal, lateral and vertical velocities of the UAV. The three are PD-like controllers, and send velocity commands. The reference to follow is the center of the image for both, the lateral and the longitudinal controllers to keep it in the center of the helipad, and in the case of the vertical controller is to approximate to zero meters of altitude. To increase the safety of the action of the vertical controller it is conditioned by the current error of the other two controllers, it means that the vertical controller only acts when the lateral and longitudinal errors are under a predefined threshold.

A set of tests were done with good results. Here it is presented one of these tests. Figure 1 shows the $3 \mathrm{D}$ reconstruction of the flight using the GPS data. Figure 2 shows the measured done using the 3D positioning based on the homography estimation for longitudinal (pitch), lateral (roll), and vertical (altitude) axis. In this flight the autonomous landing starts at 4 meters. The RMSE value for the longitudinal position in this experiment is 0.5254 meters and for the lateral position is 0.6817 .

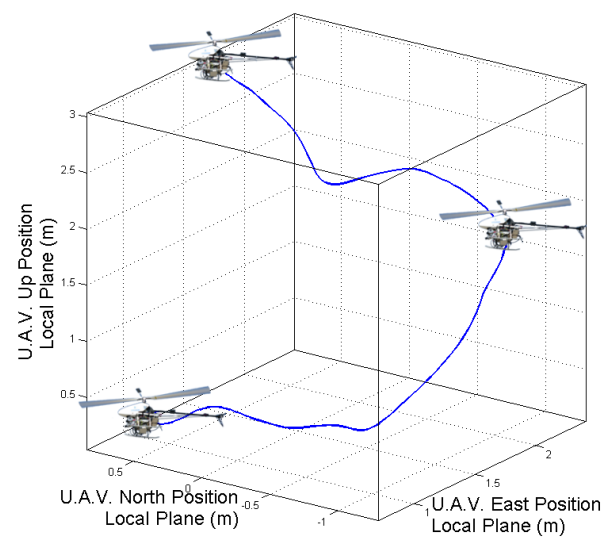

Figure 1: 3D flight reconstruction of third test of fully autonomous landing.

More details related to this work are available in these author's previous publications [25], [26].

\subsection{Object Following/Inspection}

The autonomous navigation of a UAV is a complex task that depends also on the environment, 


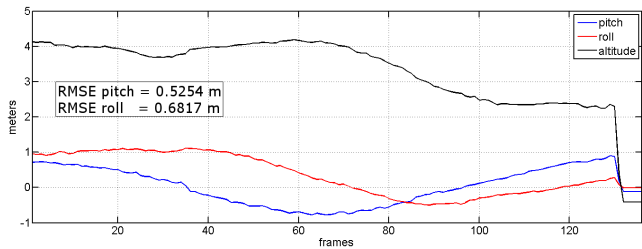

Figure 2: Homography estimation for pitch, roll and altitude of third test of fully autonomous landing.

previously it was presented the control system approach of the lateral, longitudinal and vertical velocities of a helicopter and their application for the autonomous landing task. Here it is presented the control approach of the longitudinal and the heading velocities of a quadrotor, and its application for object following or inspection. In this case we follow a helium balloon guided with a cord. The UAV must keep the balloon always in the center of the image and from a safety distance to avoid any collision. The target is detected by a predefined color. The color will be tracked along the image sequence. The tracking is performed by using the Continuously Adaptive Mean Shift [27] (CamShift). This algorithm is based on the mean shift originally introduced by Fukunaga and Hostetler [28]. This algorithm accounts for the dynamic nature of changes in lighting conditions by dynamically adapting to changes in probability distributions of color. The size and center of this color object can be obtained from real-time images via the CamShift algorithm operating on the adjusted color probability distribution.

The system in this case is composed by an AscTec Pelican quadrotor, with looking forward camera. The control approach is composed by two PD-like Fuzzy controllers. The longitudinal velocity controller try to keep the quadrotor flying from a safe distance to the object to track, following it in case that the object is moving far away from the UAV. The second Fuzzy controller manage the orientation (heading) of the UAV by velocity commands to keep the object in the center of the image, respect to the horizontal axis, turning left or right in case it is necessary.

Figure 3 shows the trajectory that was made by the UAV and some captions from the onboard camera. In this case a red balloon was followed during almost two minutes. The initial point of the aircraft is marked with the number 1 . Then the aircraft follows the trajectory marked by the consecutive numbers.

Figure 4 shows the measurement of the balloon's size during the test. The size of the balloon gives the information to the controller to go ahead, when the object it is far away or to go back when it is so near. Some captions of the most representing movements are included in this Figure. The first picture represents the beginning of the test before the motors ignition. The second one shows the normal situa-

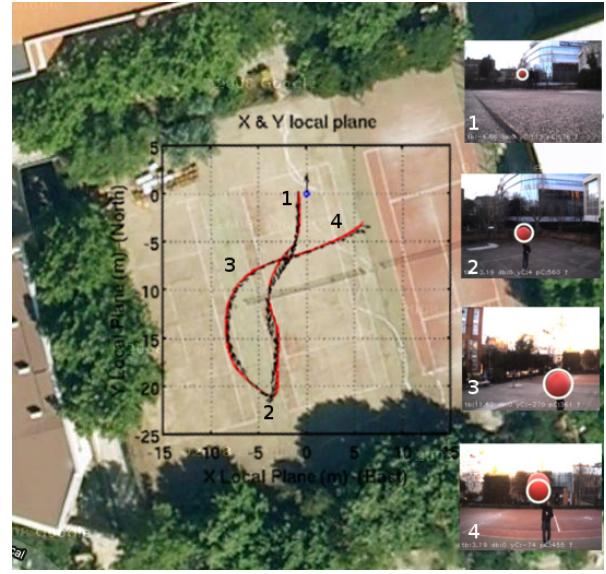

Figure 3: Trajectory of the Pelican-UAV during the First Aerial Object Following Test.

tion where the object has the predefined size. To test the reverse movements of the aircraft, the balloon was moved against the UAV, that is shown on the third picture. The fourth picture shows how the UAV was recovered from the previous situation in few seconds. This test was performed with a RMSE of 18.8495 pixels for the longitudinal velocity controller.

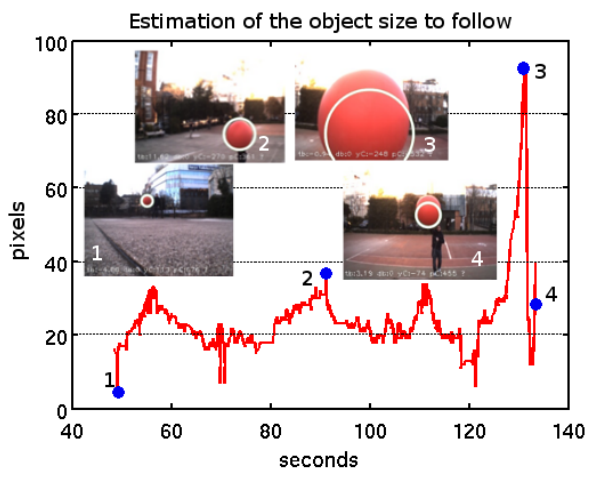

Figure 4: Measurements for the distance between the UAV and the aerial object

The Figure 5 shows the estimation of the angle between the red balloon, the UAV and the center of the image. This measurement is obtained by the visual algorithm, and is used as input for the heading controller. As it is shown in this Figure, the controller keeps the object in the center of the image, despite the effects of the wind and the random trajectory. Some captions of the most critical moments are included in the Figure. This test was performed with a RMSE of 9.3198 degrees for the orientation controller.

More details related to this work are available in this author's previous publication [29].

\subsection{Avoiding Collisions}

The avoidance task aims to keep the target in the image plane at constant bearing, either right or left 


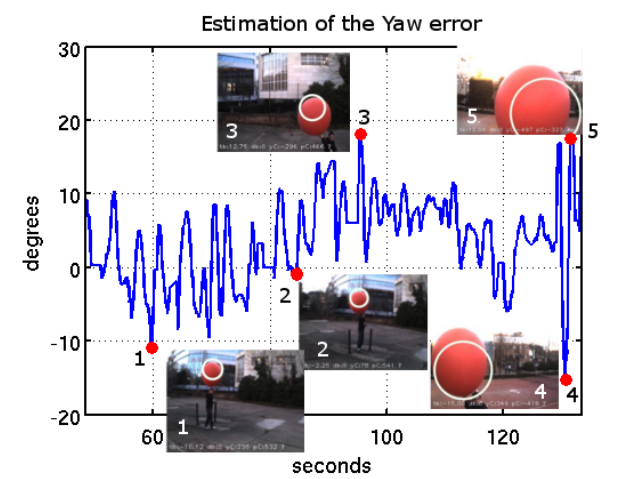

Figure 5: Measurements for the orientation of the UAV

(as seen from image centre). When the object is first detected it is pushed to the edge of the image (far left or right side), and kept at a fixed position that represents a constant relative bearing. Flight tests were conducted using Parrot-AR.Drone platform. Visual awareness is achieved by using an onboard forward-looking camera. Images from the camera are then sent for off-board processing in a laptop ground-station. The outcome of the visual processing (and control commands) are then sent back to the vehicle using a WiFi link. Communication routines were developed to send and receive information from the vehicle. A typical orange traffic cone was selected as the object to avoid. We used a motion capture system to record accurately the trajectory of vehicle with the maximum precision. This information was used for 3D plotting, and no data was used for the control of the aircraft. The position of the quadcopter is calibrated at the beginning of the test, being the initial position the point $(0,0,0)$ meters. The obstacle to avoid is located in front of the initial position of the quadcopter at distance of 6 meters and at 1.1 meters from the floor $(5,0,1.1)$. A $3 \mathrm{D}$ reconstruction of the flight is shown in Figure 6 .

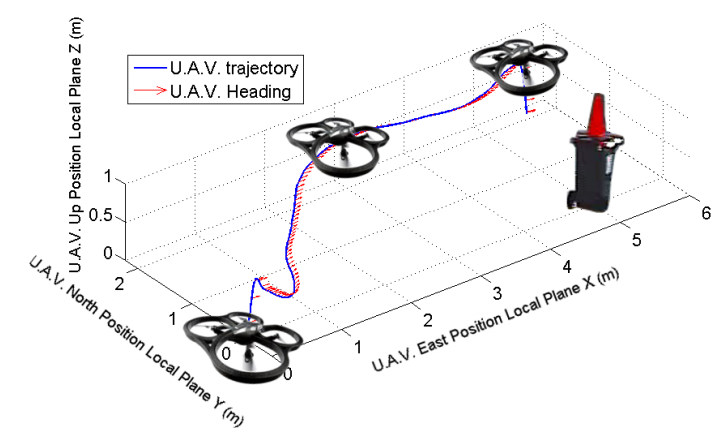

Figure 6: 3D flight reconstruction of the flight path. The obstacle to avoid is a orange traffic cone located at the position $(5,0,1.1)$.

The behavior of the controller is represented in the Figure 7 which shows the evolution of the error during the test. The red line step represents the moment in which the image processing starts. The measure of the step is 25 degrees, but at the moment when the step is applied the aircraft was looking at the opposite side increasing the step command to 35 degrees. To evaluate the behavior of the controller we use the error estimator of the root mean-square error (RMSE). The lower value this error estimator of $R M S E=9.57$ degrees. The quick response of the controller shown in this Figure corroborates the good performance of the optimized-controller.

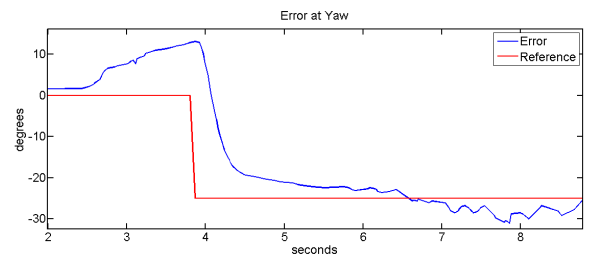

Figure 7: Evolution of the error during a real test.

More details related to this work are available in this author's previous publication [30].

\section{Conclusions and Future Work}

In this work it is proposed the use of vision based Fuzzy control technique for robotics applications, with special mention to the Unmanned Aerial Vehicles (UAVs). It was explained the advantages of using a vision sensor, as well as the uses of Fuzzy control techniques for this purpose. Furthermore, this paper presented a methodology to design, develop and adjust vision based Fuzzy control approaches for general purposes in robotics. It was explained how to use a developed Open source software tool under the Robotics Operative System (ROS) framework to design, implement and tune Fuzzy controllers. Furthermore, three different vision based Fuzzy control approaches for autonomous navigation of UAVs were shown to validate the proposed methodology and system.

For future work, the authors are working on an extension of the capabilities of the developed software and developing new approaches for other UAVs applications.

\section{Acknowledgments}

The work reported in this paper is the result of several research stages at the Computer Vision Group Universidad Politécnica de Madrid and at the Australian Research Centre for Aerospace Automation - Queensland University of Technology (ARCAAQUT). The authors would like to thank Ivan F. Mondragón for his constant help during all these years and experiments, and Luis Mejías for his collaboration during the authors' stay at the ARCAAQUT in Australia. This work has been sponsored by the Spanish Science and Technology Ministry under the grant CICYT DPI2010-20751-C02-01 and 
by the IRSES Program Marie Curie FP7 - PIRSESGA-2009-230797 - ICPUAS (International Cooperation Program for Unmanned Aerial Systems Research and Development).

\section{References}

[1] Y. Shirai and H. Inoue. Guiding a robot by visual feedback in assembling tasks. Pattern Recognition, 5(2):99 - 108, 1973.

[2] A. C. Sanderson and L. E. Weiss. Adaptative visual servo control of robots. In Robot Vision (A. Pugh, ed), pages 107-116, 1983.

[3] A. Isidori, L. Marconi, and A. Serrani. Robust nonlinear motion control of a helicopter. Automatic Control, IEEE Transactions on, 48(3):413 - 426, mar 2003.

[4] Hebertt Sira-Ramirez, Rafael Castro-Linares, and Eduardo Liceaga-Castro. A liouvillian systems approach for the trajectory planningbased control of helicopter models. International Journal of Robust and Nonlinear Control, 10(4):301-320, 2000.

[5] E. Frazzoli, M.A. Dahleh, and E. Feron. Realtime motion planning for agile autonomous vehicles. In American Control Conference, 2001. Proceedings of the 2001, volume 1, pages 43-49 vol.1, 2001.

[6] E. Frazzoli, M.A. Dahleh, and E. Feron. Trajectory tracking control design for autonomous helicopters using a backstepping algorithm. In American Control Conference, 2000. Proceedings of the 2000, volume 6, pages $4102-4107$ vol.6, 2000.

[7] Omid Shakernia, Yi Ma, T. John Koo, T. John, and Shankar Sastry. Landing an unmanned air vehicle: Vision based motion estimation and nonlinear control. Asian Journal of Control, 1:128-145, 1999.

[8] S. Saripalli, J.F. Montgomery, and G.S. Sukhatme. Vision-based autonomous landing of an unmanned aerial vehicle. In Robotics and Automation, 2002. Proceedings. ICRA '02. IEEE International Conference on, volume 3, pages $2799-2804,2002$.

[9] Takeshi Yamakawa. Stabilization of an inverted pendulum by a high-speed fuzzy logic controller hardware system. Fuzzy Sets and Systems, 32(2):161 - 180, 1989. Applications of Fuzzy Logic Control to Industry.

[10] Kevin M. Passino and Stephen Yurkovich. Fuzzy Control. Addison-Wesley Longman Publishing Co., Inc., Boston, MA, USA, 1st edition, 1997.

[11] T. Takagi and M. Sugeno. Fuzzy identification of systems and its applications to modeling and control. Systems, Man and Cybernetics, IEEE Transactions on, SMC-15(1):116132, Jan 1985.
[12] Kok-Meng Lee and Yifei Qian. A vision-guided fuzzy logic control system for dynamic pursuit of a moving target. Microprocessors and Microsystems, 21(9):571 - 580, 1998.

[13] Li Yang, Zhiqiang Cao, Min Tan, and Guoping Liu. Vision-based fuzzy coordination control for multiple robots. In Robotics, Automation and Mechatronics, 2008 IEEE Conference on, pages 521-524, Sept 2008.

[14] Hyun il Kwon, Jaehong Park, Wonsang Hwang, Jong hyeon Kim, Chang hun Lee, M.L. Anjum, Kwang soo Kim, and Dong il Cho. Sensor data fusion using fuzzy control for vor-based vision tracking system. In Intelligent Robots and Systems (IROS), 2010 IEEE/RSJ International Conference on, pages 2920-2925, Oct 2010.

[15] J.W. Tweedale. Fuzzy control loop in an autonomous landing system for unmanned air vehicles. In Fuzzy Systems (FUZZ-IEEE), 2012 IEEE International Conference on, pages 1-8, June 2012.

[16] Ashraf Qadir, William Semke, and Jeremiah Neubert. Vision based neuro-fuzzy controller for a two axes gimbal system with small UAV. Journal of Intelligent and Robotic Systems, 74(3-4):1029-1047, 2014.

[17] Olivares-mendez snt homepage, 2014.

[18] Miguel A. Olivares-Mendez, Ivan Mondragon, Pascual Campoy, , Carol Martinez, and J. F. Correa. Fuzzy control system navigation using priority areas. In Computational Intelligence in Decision and Control, 2008, pages 987-996, 2008.

[19] Miguel A. Olivares-Mendez, Ivan Mondragon, Pascual Campoy, , and Carol Martinez. Nonsymmetric membership function for fuzzybased visual servoing on-board a uav. In $\mathrm{Com}$ putational Intelligence in Foundations and Applications, 2010, pages 300-307, 2010.

[20] Iván Mondragón, Miguel Olivares-Méndez, Pascual Campoy, Carol Martínez, and Luís Mejias. Unmanned aerial vehicles uavs attitude, height, motion estimation and control using visual systems. Autonomous Robots, 29:1734, 2010. 10.1007/s10514-010-9183-2.

[21] Miguel Olivares and J.A.F. Madrigal. Fuzzy logic user adaptive navigation control system for mobile robots in unknown environments. Intelligent Signal Processing, 200\%. WISP 200\%. IEEE International Symposium on, pages 1-6, Oct. 2007.

[22] Bouguet Jean Yves. Pyramidal implementation of the lucas-kanade feature tracker. Technical report, Intel Corporation. Microprocessor Research Labs, Santa Clara, CA 95052, 1999.

[23] Jianbo Shi and Carlo Tomasi. Good features to track. In 1994 IEEE Conference on Computer Vision and Pattern Recognition (CVPR'94), pages 593-600, 1994. 
[24] M. A. Fischer and R. C. Bolles. Random sample concensus: a paradigm for model fitting with applications to image analysis and automated cartography. Communications of the ACM, 24(6):381-395, 1981.

[25] M.A. Olivares-Mendez, I.F. Mondragon, P. Campoy, and C. Martinez. Fuzzy controller for uav-landing task using 3d-position visual estimation. In Fuzzy Systems (FUZZ), 2010 IEEE International Conference on, pages 1-8, July 2010.

[26] Miguel A. Olivares-Mendez, Ivan Mondragon, and Pascual Campoy Cervera. Autonomous landing of an unmanned aerial vehicle using image-based fuzzy control. In Second Workshop on Research, Development and Education on Unmanned Aerial Systems (RED-UAS 2013), 2013.

[27] Gary R. Bradski. Computer vision face tracking for use in a perceptual user interface. Intel Technology Journal, 1(Q2), 1998.

[28] K. Fukunaga and L. Hostetler. The estimation of the gradient of a density function, with applications in pattern recognition. Information Theory, IEEE Transactions on, 21(1):32 - 40, January 1975.

[29] Miguel A. Olivares-Mendez, Ivan Mondragon, Pascual Campoy Cervera, Luis Mejias, and Carol Martinez. Aerial object following using visual fuzzy servoing. In First Workshop on Research, Development and Education on Unmanned Aerial Systems (RED-UAS 2011), 2011.

[30] Miguel A. Olivares-Mendez, Luis Mejias, Pascual Campoy, and Ignacio Mellado-Bataller. Quadcopter see and avoid using a fuzzy controller. In World Scientific Proceedings Series on Computer Engineering and Information Science, 2012, 2012. 